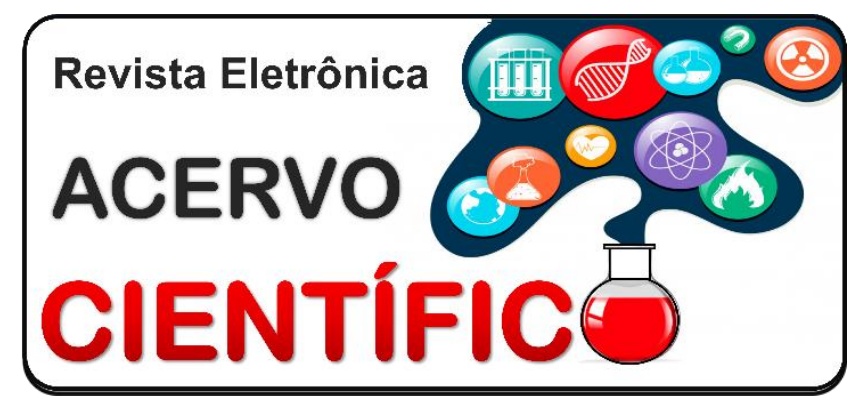

REVISÃO BIBLIOGRÁFICA

Recebido em: 7/2020

Aceito em: 8/2020

Publicado em: 10/2020

\title{
O acesso aos serviços de saúde da população ribeirinha: um olhar sobre as dificuldades enfrentadas
}

\author{
Access to the health services of the ribeirinha population: a look at the difficulties faced \\ Acceso a los servicios de salud de la población ribeirinha: una mirada a las dificultades \\ enfrentadas
}

Adilson Mendes de Figueiredo Júnior ${ }^{1 *}$, Glória Letícia Oliveira Gonçalves Lima1 ${ }^{1}$, Karla Ariana Dias Vilela $^{1}$, Eliane Conceição da Costa ${ }^{1}$, Maria Lúcia Costa dos Santos ${ }^{1}$, Maria da Conceição Nascimento Freitas ${ }^{2}$, Yasmin Martins de Sousa ${ }^{2}$, Fábio da Costa Ferreira ${ }^{3}$, Christian Boaventura dos Santos ${ }^{3}$, Cristiane de Oliveira Calandrini ${ }^{4}$.

Resumo: Esse artigo buscou discutir o acesso da população ribeirinha aos serviços públicos de saúde. Um importante desafio para o SUS atualmente é alcançar as populações mais prejudicadas, incluindo as comunidades ribeirinhas e as demais populações interiorizadas. Tratou-se de um estudo de natureza qualitativo-descritiva sob método de revisão bibliográfica. A igualdade no acesso é algo que se deixa a desejar, uma vez que pessoas que residem em metrópoles e grandes centros urbanos possuem maior aproximação e facilitação no acesso a saúde, já os ribeirinhos muita das vezes, precisam se deslocar de onde reside para outra comunidade, cidade e até mesmo outro município, acarretando gastos extras na renda familiar ou até mesmo impedindo aquele cidadão de buscar o sistema de saúde. Quando se trata de avanço em saúde, a educação caminha ao lado do ciclo evolutivo de um indivíduo, por meio desta que o ser humano é capaz de aprender e desenvolver atividades.

Palavras-chave: Enfermagem, Educação em saúde, Grupos étnicos.

\begin{abstract}
This article sought to discuss the access of the riverside population to health services. An important challenge for SUS nowadays is to reach the most affected populations, including the riverside communities and other inland populations. It was a qualitative-descriptive study using a literature review method. Equal access is something to be desired, since people who live in cities and large urban centers have a closer relationship and facilitate access to health care, while riverside residents often need to move from where they live to another community, city and even another municipality, leading to extra expenses on family income or even preventing that citizen from seeking health care. When it comes to advancement in health, education goes hand in hand with an individual's evolutionary cycle, through which human beings are able to learn and develop activities.
\end{abstract}

Keywords: Nursing, Health education, Ethnic groups.

\footnotetext{
1 Escola Superior da Amazônia (ESAMAZ), Belém - PA. *E-mail: adilsonmdfj@hotmail.com

2 Universidade do Estado do Pará (UEPA), Belém - PA.

3 Universidade Federal do Pará (UFPA), Belém - PA.

${ }^{4}$ Hospital Naval de Belém (HNBe), Belém - PA.
} 
Resumen: Este artículo buscaba discutir el acceso de la población ribereña a los servicios de salud. Un desafío importante para el SUS hoy en día es llegar a las poblaciones más afectadas, incluidas las comunidades ribereñas y otras poblaciones del interior. Fue un estudio cualitativo descriptivo que utilizó un método de revisión de literatura. El acceso equitativo es algo deseable, ya que las personas que viven en ciudades y grandes centros urbanos tienen una relación más estrecha y facilitan el acceso a la atención médica, mientras que los residentes de la ribera a menudo necesitan mudarse de donde viven a otra comunidad, ciudad e incluso otro municipio, lo que genera gastos adicionales en el ingreso familiar o incluso impide que ese ciudadano busque atención médica. Cuando se trata de avanzar en la salud, la educación va de la mano con el ciclo evolutivo de un individuo, a través del cual los seres humanos pueden aprender y desarrollar actividades.

Palabras clave: Enfermería, Educación para la salud, Grupos étnicos.

\section{INTRODUÇÃO}

Um importante desafio para o Sistema Único de Saúde (SUS) atualmente é alcançar as populações mais prejudicadas, incluindo as comunidades ribeirinhas que são aquelas caracterizadas como população tradicionais e residentes das margens dos risos e populações interiorizadas. Nesse sentido, pretende-se levar ideias estratégicas para redução dessa carência de acesso aos serviços de saúde (BRASIL GB, et al., 2016).

A igualdade no acesso é algo que se deixa a desejar, uma vez que pessoas que residem em metrópoles e grandes centros urbanos possuem maior aproximação e facilitação no acesso a saúde, já os ribeirinhos muita das vezes, precisam se deslocar de onde reside para outra comunidade, cidade e até mesmo outro município, acarretando gastos extras na renda familiar ou até mesmo impedindo aquele cidadão de buscar o sistema de saúde, por falta de condições financeiras, isso é causado pela falta de cobertura que a saúde deveria proporcionar para essa população (BRASIL GB, et al., 2016).

A precariedade ao acesso dos ribeirinhos aos serviços de saúde pública revela a necessidade de medidas amplas de promoção de saúde, associada à oferta de serviços adequada à realidade das comunidades rurais ribeirinhas distantes dos espaços urbanos (FRANCO EC, et al., 2015).

Segundo Oliveira JWD, et al. (2012), a legislação que envolve a Atenção Primária em Saúde (APS) configura uma forma de valorização da assistência prestada pelo SUS dentro da inclusão de pessoas socialmente afastados, entende-se que é por meio da Estratégia Saúde da Família (ESF) que devam ocorrer os primeiros contatos das populações mais afastadas com o sistema de saúde, ou seja, é conceituada como a porta de entrada e de apresentação dos serviços públicos de saúde e do entendimento das necessidades dos povos nativos assim como seus problemas

O conhecimento das características que envolvem a Atenção Primária em Saúde significa uma relação muito mais duradoura entre usuários e profissionais de saúde, pois, dessa forma, se estabelece um vínculo que auxilia no progresso da relação terapêutica contínua entre os envolvidos, um acompanhamento multiprofissional de diversas áreas da saúde (OLIVEIRA JWD, et al., 2012). Assim, os moradores dessas comunidades, mesmo vivendo em áreas geográficas próximas aos centros urbanos, ainda encontram diversas dificuldades para acessarem os serviços de saúde, principalmente, pelas características residenciais, como casas presentes em margens de rios, no interior de florestas e habitações com difícil acesso para profissionais de saúde e a qualquer rede de atenção primária, secundária e terciária (BRASIL GB, et al., 2016).

Partindo destas questões que atravessam o modo de vida dos ribeirinhos, foi possível identificar desafios diversificados para as equipes de saúde que os assistem, no sentido de ofertar mais saúde a essa população, apesar de considerar relacionados à baixa densidade demográfica. Esse cenário remete às dificuldades operacionais para 0 trabalho ser desenvolvido, pela indisponibilidade de transporte para oportuno deslocamento da equipe de saúde, na baixa frequência de contato com os ribeirinhos. Dessa 
forma é possível vislumbrar a influência significativa no eixo principal do cuidado com esta população (MOTISUKI D, et al., 2018).

Neste cenário é essencial que o cuidado adote uma visão holística da população, entendendo-se que o vínculo criado se torna um elo, onde a população ribeirinha cria uma maior confiança e credibilidade nos profissionais de saúde envolvidos na assistência. A enfermagem nesse contexto é de suma importância, pois representa a porta de entrada a esses serviços, é o primeiro contato com o indivíduo na questão do acolhimento e no esclarecimento de informações (VELASQUES CL, et al., 1986).

Em diferentes casos, o problema / doença é resolvido, mas outras informações que deveriam ser repassadas a essas pessoas não são compartilhadas, ou seja, faltam atividades educacionais que possam mostrar aos profissionais envolvidos a importância da cultura em saúde (SANTANA FA, 2011).

Portanto, este trabalho tem como vertente o Art. 196 da Constituição Federal de 88, que objetiva a saúde sendo direito de todos e dever do estado, de modo que deve assegurar à saúde para essa população pouco visada, garantindo a saúde com integralidade, de forma igualitária e de acesso universal para esses ribeirinhos. O objetivo do atual estudo foi descrever através de uma revisão da literatura, o acesso da população ribeirinha aos serviços de saúde.

\section{MÉTODOS}

Tratou-se de um estudo de natureza qualitativo-descritiva sob método de revisão bibliográfica desenvolvida por meio do método da revisão integrativa da literatura, cujo objetivo foi proporcionar a incorporação das evidências de estudos a partir do tema "O acesso aos serviços de saúde da população ribeirinha: um olhar sobre as dificuldades enfrentadas" para que se constitua um importante elo na promoção das interações, para busca das melhores estratégias e políticas que possibilite um cuidado holístico aos profissionais envolvidos.

Durante o processo de busca dos artigos, utilizaram-se os descritores Enfermagem; Educação em Saúde e Grupos Étnicos conjuntamente. Foi achado um total de 487 artigos que após a utilização dos filtros "disponível", "artigo" e "2012-2019", chegou-se a 74 manuscritos e após a aplicação dos critérios de inclusão e exclusão o total final foi de 7 artigos (Figura 1).

Figura 1 - Busca bibliográfica com utilização dos filtros.

Descritores: Enfermagem AND Educação em Saúde AND Grupos Étnicos

Fonte: Figueiredo Júnior AM, et al., 2020.

Total: 487 ARTIGOS

Os artigos foram selecionados e analisados, de acordo com o tema proposto. Após a seleção dos artigos indexados, realizou-se uma avaliação e análise do material obtido, para selecionar o que é de interesse 
para as pesquisadoras, em seguida foi realizada uma leitura minuciosa, a fim de não serem perdidos aspectos importantes para o enriquecimento de estudo e confecção da redação final da pesquisa.

Como critérios de inclusão foram utilizados artigos completos que contemplem o tema e objetivo da pesquisa, que tenha relevância ao tema proposto do projeto da pesquisa dentre os anos de 2012 a 2019 , produzidos no Brasil e em português. Como critérios de exclusão foram excluídos artigos que necessitem que alguma taxa de pagamento para serem lidos e artigos que relacionem outros temas além de Assistência aos Ribeirinhos.

Os riscos da pesquisa foram mínimos, pois tratou-se de uma revisão bibliográfica, tais riscos foram representados por uma possibilidade de troca de informações e seus respectivos autores durante a discussão do projeto, para minimizar tais possibilidades todos os artigos científicos foram organizados em pastas e lidos individualmente para não haver conflito de idéias que pudessem confundir os pesquisadores.

Com os resultados do estudo, visa-se contribuir para incrementar os conhecimentos a respeito da assistência às populações ribeirinhas. Espera-se também que ocorra melhoria das ações desenvolvidas pelos profissionais da área da saúde, mais especificamente os enfermeiros que atuam na área de atendimento a esse público.

\section{RESULTADOS}

Optou-se em organizar os resultados de acordo com o título dos artigos, o estado onde foram publicados, ano de publicação e objetivo dos estudos (Tabela 1). Respeitou-se a totalidade dos achados científicos e organizaram-se tabelas caracterizadoras para melhor observação e entendimento de cada artigo científico. Durante a pesquisa foram achados 7 artigos que variaram entre os anos de 2012 a 2018, abordavam os aspectos gerais relacionados a assistência à saúde aos Ribeirinhos.

De acordo com o resultado das pesquisas encontradas nesta pesquisa, observa-se a seguir a Tabela 2 que destaca por região a produção científica sobre o acesso da população ribeirinha aos serviços de saúde. Verificam-se na Tabela 2 que as regiões com maior incidência de pesquisa sobre o acesso da população ribeirinha aos serviços de saúde dentre os anos de 2012 a 2018, a região Norte prevaleceu com $71,40 \%$ do conteúdo de pesquisas, já a região Sudeste apresenta $28,60 \%$ dos artigos pesquisados. 
Tabela 1 - Características dos artigos científicos que abordam o acesso da população ribeirinha aos serviços de saúde.

\begin{tabular}{|c|c|c|c|c|}
\hline Ano & Autores & Local & Título & Objetivo \\
\hline 2012 & $\begin{array}{l}\text { MAIA SMS, } \\
\text { SILVA LR }\end{array}$ & RJ & $\begin{array}{l}\text { Saberes e práticas de mães } \\
\text { ribeirinhas e o cuidado dos filhos } \\
\text { recém-nascidos: contribuição para } \\
\text { a enfermagem }\end{array}$ & $\begin{array}{l}\text { Identificar os valores culturais } \\
\text { maternos no cuidado dos filhos } \\
\text { recém-nascidos ribeirinhos. }\end{array}$ \\
\hline 2015 & $\begin{array}{l}\text { FRANCO } \\
\text { EC, et al. }\end{array}$ & SP & $\begin{array}{l}\text { Promoção da saúde da população } \\
\text { ribeirinha da região amazônica: } \\
\text { relato de experiência }\end{array}$ & $\begin{array}{l}\text { Relatar as ações de promoção de } \\
\text { saúde realizadas em comunidades } \\
\text { ribeirinhas pelas equipes } \\
\text { expedicionárias do projeto "FOB- } \\
\text { USP em Rondônia". }\end{array}$ \\
\hline 2016 & $\begin{array}{l}\text { BRASIL GB, } \\
\text { et al. }\end{array}$ & PA & $\begin{array}{l}\text { Modo de vida ribeirinho e a } \\
\text { longitudinalidade do cuidado na } \\
\text { atenção primária em saúde }\end{array}$ & $\begin{array}{l}\text { Identificar atitudes que são } \\
\text { valorizadas pelos profissionais das } \\
\text { equipes da Estratégia Saúde da } \\
\text { Família Ribeirinhas relacionadas à } \\
\text { longitudinalidade do cuidado. }\end{array}$ \\
\hline 2017 & $\begin{array}{l}\text { MIRANDA } \\
\text { HR, et al. }\end{array}$ & PA & $\begin{array}{l}\text { Desvendando saberes e } \\
\text { preocupações sobre a saúde entre } \\
\text { homens ribeirinhos }\end{array}$ & $\begin{array}{l}\text { Identificar os saberes de homens } \\
\text { ribeirinhos sobre a saúde. }\end{array}$ \\
\hline 2018 & $\begin{array}{l}\text { QUEIROZ } \\
\text { MKS, et al. }\end{array}$ & PA & $\begin{array}{l}\text { Fluxos assistenciais e a } \\
\text { integralidade da assistência } \\
\text { saúde de ribeirinhos }\end{array}$ & $\begin{array}{l}\text { Verificar se a organização da } \\
\text { atenção em saúde vigente na llha do } \\
\text { Combú atende ao princípio da } \\
\text { integralidade da assistência à saúde } \\
\text { de sua população }\end{array}$ \\
\hline 2018 & $\begin{array}{l}\text { MOTISUKI } \\
\text { D, et al. }\end{array}$ & PA & $\begin{array}{l}\text { Roda de conversa como estratégia } \\
\text { de educação em saúde para a } \\
\text { enfermagem }\end{array}$ & \begin{tabular}{lrrr}
\multicolumn{3}{l}{ Demonstrar a relevância da roda de } \\
conversa & como & estratégia & para \\
educação & em & saúde & em \\
enfermagem. & & &
\end{tabular} \\
\hline 2018 & $\begin{array}{l}\text { GAMA ASM, } \\
\text { et al. }\end{array}$ & $A M$ & $\begin{array}{ll}\text { Inquérito de saúde } & \text { em } \\
\text { comunidades ribeirinhas } & \text { do } \\
\text { Amazonas, Brasil. } & \end{array}$ & $\begin{array}{l}\text { Apresentar os principais aspectos } \\
\text { metodológicos e descrever as } \\
\text { características socioeconômicas, } \\
\text { demográficas e de saúde dos } \\
\text { ribeirinhos de Coari, Amazonas, } \\
\text { Brasil. }\end{array}$ \\
\hline
\end{tabular}

Fonte: Figueiredo Júnior AM, et al., 2020.

Tabela 2 - Características dos artigos científicos que abordam o acesso da população ribeirinha aos serviços de saúde.

\begin{tabular}{ccc}
\hline \multicolumn{3}{c}{ Artigos científicos por regiões no Brasil } \\
\hline Regiões & Pesquisas & $(\%)$ \\
\hline Norte & 5 & $71,40 \%$ \\
Nordeste & 0 & $0,00 \%$ \\
Sul & 0 & $0,00 \%$ \\
Sudeste & 2 & $28,60 \%$ \\
Centro-oeste & 0 & $0,00 \%$ \\
\hline Total & 7 & $100,00 \%$
\end{tabular}

Fonte: Figueiredo Júnior AM, et al., 2020.

\section{DISCUSSÃO}

Um importante desafio para a Saúde Pública na atualidade é o alcance das populações mais afastadas, dentre elas, as comunidades ribeirinhas e quilombolas, muito expressivas na região amazônica, de modo especial no Estado do Pará. Nesse sentido é necessária a criação de estratégias para redução das 
desigualdades no acesso aos serviços de saúde com políticas de equidade e universalidade, na perspectiva de atender as demandas de saúde desses povos (BRASIL GB, et al., 2016).

O conceito de saúde pode ser determinado por inúmeros fatores, que são formados por 4 pilares: a biologia humana, o ambiente, o estilo de vida e o sistema de atenção à saúde. $O$ estilo de vida é um dos elementos mais determinantes da promoção da saúde e no processo saúde $\mathrm{x}$ doença e extremamente significante no processo de construção de uma qualidade de vida saudável. A adoção de hábitos saudáveis de vida, como atividade física e alimentação balanceada, é essencial para o enfrentamento de condições ou situações adversas tanto do meio ambiente quanto do meio social (SILVA DMPP e TOMANIK EA, 2012).

A saúde ribeirinha é dada mediante a Política Nacional de Atenção Básica, implementada no país, pelas Portarias MS/GM no 2.488 e 2.490, voltadas para a implantação e operacionalização das atividades de saúde, bem as Equipes de Saúde das Famílias Ribeirinhas (ESFR) e Unidades Básicas de Saúde Fluviais (UBSF), sobretudo na região amazônica (FRANCO EC, et al., 2015).

As políticas de saúde no Brasil avançaram no campo políticas sociais, para adoção do Sistema Único de Saúde (SUS) com seus princípios de universalidade, integralidade, equidade, descentralização e participação popular. Assim, foi possível a introdução de práticas em saúde que permitem a quebra das formas tradicionais de assistência à saúde e de efetivação do cuidado. Profissionais de diferentes áreas do conhecimento, como médicos e enfermeiros, deram início ao fortalecimento dos conceitos de saúde universal no país (DIAS GR, et al., 2018).

Deve-se considerar o dimensionamento do Brasil, onde é possível encontrar populações não apenas residentes em áreas urbanas e rurais, mas às margens de rios, as denominadas comunidades ribeirinhas. $A$ população ribeirinha é caracterizada como aquela que vive às margens dos rios. Os ribeirinhos estão isolados não apenas da cultura geral, como do acesso à mídia e alguns meios de prevenção de doenças. Em termos interacionais, o rio atua como uma estrada, criando e restringindo as possibilidades de interação, principalmente entre as crianças (FRANCO EC, et al., 2015).

O nível de saúde de uma população pode ser avaliada por diversos indicadores influentes no processo saúde-doença. A análise de indicadores de saúde, como de mortalidade e de morbidade em determinada população, é determinante para verificar as desigualdades sociais que permitem, muitas vezes, 0 aparecimento de surtos e epidemias, assim pontua-se a importância de subsídios para o planejamento em saúde (SILVA DMPP e TOMANIK EA, 2012).

O fortalecimento da Atenção Primária em Saúde (APS) caracteriza-se como uma possibilidade de inclusão dessas pessoas e, posteriormente, melhoria na qualidade de vida, tendo em vista que é por meio da Estratégia Saúde da Família (ESF) que deve ocorrer o primeiro contato do indivíduo com o sistema de saúde. Assim percebe-se que a porta de entrada da rede de serviços para atender suas necessidades torna-se essencial para o equilíbrio das relações de saúde (BRASIL GB, et al., 2016).

No contexto de assistência em saúde às populações ribeirinhas é necessário democratizar o processo de trabalho na organização dos serviços, respeitando as necessidades sociais e, principalmente, a atuação das equipes multiprofissionais, incorporando a renovação das práticas de saúde numa perspectiva holística e respeitosa em que a valorização da atenção à saúde e do cuidado possam integrar todas as características que formam essas comunidades (QUEIROZ MKS, et al., 2018).

O cotidiano dos ribeirinhos às margens dos rios é marcado pela dinâmica das águas (cheias e vazantes) e, tal situação, impõe limitações multidimensionais e em todos os sentidos. A economia encontra-se alicerçada nas atividades de pesca e extrativismo vegetal e a cultura fortemente atrelada aos povos indígenas, com hábitos alimentares diferenciados e terapia através de plantas medicinais. Os ribeirinhos, de modo geral, vivem de maneira simples com infraestrutura mínima de saneamento básico, energia elétrica e serviços de saúde. Tais características são fatores diretamente ligados ao aparecimento de doenças infecto-parasitárias, por exemplo. A assistência à saúde é centralizada na zona urbana dos municípios, com ações esporádicas dos profissionais de saúde nas comunidades, o que acaba afastando essa população do conhecimento de métodos preventivos (GAMA ASM, et al., 2018). 
Os profissionais da saúde necessitam identificar os desafios peculiares existentes nessas comunidades, principalmente, pela dispersão dos moradores na floresta ao longo do curso dos rios. O difícil acesso e essas comunidades representam grandes dificuldades operacionais para o trabalho, pela indisponibilidade de transporte e na baixa frequência de contato da equipe de saúde com os moradores. Dessa forma, devese criar meio de se efetivar a longitudinalidade do cuidado com esta população respeitando suas particularidades (BRASIL GB, et al., 2016).

Uma ferramenta de auxílio assistencial são os inquéritos populacionais de saúde que se constituem como instrumentos importantes para o reconhecimento de determinantes em saúde e desigualdades sociais. Tais indicadores têm o poder de viabilizar a avaliação das políticas públicas, sobretudo em grupos sociais excluídos ou pouco estudados em diferentes segmentos de saúde. No Brasil, ainda são poucas as informações epidemiológicas sobre as populações ribeirinhas da Amazônia (GAMA ASM, et al., 2018).

O processo de educação entre os ribeirinhos ocorre, preferencialmente, de forma oral, por meio de conversas informais transmitidas de geração a geração, formados por conteúdos de base afetiva, social, cultural, histórico e político, possibilitando uma gama de experiências e a perpetuação de seus costumes e de suas tradições, ao longo dos séculos, pelos mais velhos, aos mais novos (MIRANDA HR, et al., 2017).

Quanto à Enfermagem, o processo de educação em saúde popular permitiu que os enfermeiros pudessem incorporar os aspectos da subjetividade dos indivíduos, como suas características culturais e sociais, além de oferecer oportunidades para aprimorar as construções e experiências coletivas de educação e promoção a saúde. É nesse contexto que o uso da roda de conversa, por exemplo, torna-se um método de discussão que permite o aprofundamento do diálogo com base nas experiências que cada pessoa tem sobre o assunto (DIAS GR, et al., 2018).

Vários são os desafios que esses profissionais enfrentam para prestar assistência de qualidade, que envolvem tanto a distância como até ataque de animais. O papel da equipe de saúde em áreas ribeirinhas exige o desenvolvimento de habilidades técnico-científicas mais aprimoradas, como a realização de procedimentos específicos (cirúrgicos, diagnóstico e terapêutico), mas é importante que esse profissional não deixe de inserir em seu dia-a-dia a percepção cultural da perspectiva do cuidar (QUEIROZ MKS, et al., 2018).

Conhecendo e respeitando as práticas da cultura ribeirinha, os profissionais de saúde conseguem desenvolver sua assistência a comunidade evitando prejuízos na saúde e bem-estar. Deve-se orientar e respeitar o saber popular adotando medidas ativas e produtivas locais para valorização das práticas populares (MAIA SMS e SILVA LR, 2012).

A equipe de saúde que atua nas comunidades ribeirinhas deve atender a população no que lhes compete resolvendo os problemas de saúde da população. Para Queiroz MKS, et al. (2018), o imaginário do serviço de saúde ideal se desfaz, quando dependem de serviços secundários para dar continuidade ao tratamento dos usuários, pois ambos vão se deparar com a dificuldade de acesso a exames específicos, decorrente da falta de médicos especialistas, e até mesmo pela demora para marcação de exames.

Umas das dificuldades encontradas é a inclusão social, mediante a escassez de projetos e ações que almejam ampliar o conhecimento dessa população pouca assistida. Outra questão é educação em saúde que estimula $o$ autocuidado. Os ribeirinhos não se atentam para buscar prevenção, diagnostico ou tratamento de uma determinada patologia, isso é justificado pela falta de informação ou dificuldade de acesso à saúde, ou seja, se essas ferramentas fossem facilitadas o quadro mudaria (QUEIROZ MKS, et al., 2018).

Os profissionais da saúde não conseguem ter um olhar holístico frente ao paciente ribeirinho, buscando solucionar apenas as queixas do momento, não possuindo o cuidado de fazer uma investigação aprofundada, e isso consequentemente traz maiores agravos para a saúde desse cliente. É observado também que há uma deficiência na execução do processo de enfermagem: histórico, diagnostico, planejamento, implementação e avaliação, os quais trabalham com a finalidade de solucionar o problema do paciente. Contemplamos que existem pessoas que sabem o seu diagnóstico, porém, não entende do seu 
tratamento, o que contribui para evolução ou recuperação da doença, por isso fica evidenciado que transmitir informações para essa população é de grande valia.

\section{CONSIDERAÇÕES FINAIS}

Percebe-se que o foco é atentar a comunidade para a mudança da situação a qual vivem, através da inclusão nos serviços de saúde, a fim de reduzir essa carência, tais como: espera-se que os ribeirinhos possam ter uma equipe multiprofissional composta por enfermeiro, serviço social e clinico geral a cada três meses para atender a demanda no sentido de estimular e solucionar o problema/doença precoce dos fatores determinantes e condicionantes.

\section{REFERÊNCIAS}

1. BRASIL GB, et al. Modo de vida ribeirinho e a longitudinalidade do cuidado na atenção primária em saúde. Santa Maria, 2016; 42(1); 31-38.

2. DIAS GR, et al. Práticas de cuidado de enfermagem e de autocuidado para leishmaniose cutânea entre ribeirinhos, Nova Olinda do Norte, Amazonas, 2018.

3. FRANCO EC, et al. Promoção da saúde da população ribeirinha da região amazônica: relato de experiência. Rev. CEFAC, 2015; 17(5):1521-1530.

4. GAMA ASM, et al. Inquérito de saúde em comunidades ribeirinhas do Amazonas, Brasil. Cad. Saúde Pública, 2018.

5. MAIA SMS, SILVA LR. Saberes e práticas de mães ribeirinhas e o cuidado dos filhos recém-nascidos: contribuição para a enfermagem. Revista de Enfermagem Referência, 2012; 7: 131-138.

6. MIRANDA HR, et al. Desvendando saberes e preocupações sobre a saúde entre homens ribeirinhos. Rev enferm UFPE online, Recife, 2017; 11(9): 3446-53.

7. MOTISUKI D, et al. Roda de conversa como estratégia de educação em saúde para a enfermagem / Conversation wheel as education strategy in health for nursing. Revista de Pesquisa: Cuidado é Fundamental Online, 2018; 10(2): 379-384.

8. OLIVEIRA JWB, et al. Promoção da saúde na comunidade indígena Pankararu. Rev. bras. enferm., Brasília, 2012; 65(3): 437-444.

9. QUEIROZ MKS, et al. Fluxos assistenciais e a integralidade da assistência à saúde de ribeirinhos. Rev enferm UERJ, Rio de Janeiro, 2018.

10. SANTANA FA. Comunidades Ribeirinhas da Amazônia: Relato de Experiência. Revista Perspectiva Amazônia, $2011 ; 3(6): 47-58$.

11. SILVA DMPP, TOMANIK EA. Morbidade referida por moradores ribeirinhos de Porto Rico, PR, Brasil. Rev Bras Enferm, Brasília, 2012; 63(3): 452-8.

12. VELASQUES CL, et al. O enfermeiro no desenvolvimento da comunidade; atuação como educador. Rev. Bras. Enf, 1986; 39(3): 16-20. 